\title{
Research of Method of Module Division Based on AHP Analyses
}

\author{
G. Han \& G.J. Chen \& S.H. Su \& F.C. Wu \& C.Y. Zeng \\ Hangzhou, Danzi, University, ZheJiang, China
}

\begin{abstract}
The research proposes a module creation method based on AHP which analyses customers' demands weights in the process of product design. First of all, with establishing the product conceptual structure which correspond the personalized needs of customers and analyzing the similar characteristics of the component in the whole life cycle, the research forms a relationship matrix including different components; then this research analyzes the ordered clustering process of modules based on the fuzzy clustering algorithms. Compared with other optimization algorithms, the method of fuzzy clustering module based on AHP analysis can divide product in a more efficient and reasonable way, and it has a good adaptability and versatility. With using jack products as an example to verify the proposed method, this research modularizes the product in a reasonable way.
\end{abstract}

KEYWORD: AHP; Modularization; Fuzzy clustering algorithm; Customer demand

\section{GENERAL INSTRUCTIONS}

Module division is the foundation and key of the modular design. Stone invented a method of modular creation based on heuristic methods which depends on three judgment rules: dominant flow, branching flow and conversion flow to divide module (Stone et al, 2000); After Stone, F GAO created a formal guidelines and quantized model based on generalized directing graph and QFD, and he divided the module using Fuzzy clustering algorithm (Gao et al, 2007). The creation of the module now is not only simple but also quietly limited. It can't describe the modular products in a systematic and qualitative way. Thus, after decomposing the customers' needs and calculating the weight of functional unit, this article divides the module using Fuzzy clustering algorithm based on AHP analysis of customers' needs and verifies the feasibility.

\section{ESTABLISH PART RELATIONSHIP MATRIX}

\subsection{The analysis of customers' demand of multifunction hydraulic jacks products}

Functional planning is the bridge between the customers' needs and the subsequent design. The main purpose of it is to define, describe the function, to analyze the mapping relation of product features and customers' needs (Sun, 2000, Dong \& Jiang, 1991). Then it explore an abstract expression of function generating solution to satisfy the customers' needs which fulfill the task of customers' needs. Generally speaking, the customers don't know the product they purchase very well, nor do they know the demands they needs (Zhu et al. 2008). Because of the different needs of the customers, we need to further process the demands raised by the customers so that the computer recognizes the information of it and at the same time transform it into the technical rules of the product. In fact, the customers' demands are the same as different product attributes (Tan et al. 2008). Through the market, network and conservation to customers, we find out the customers' demands are mainly in the following areas.

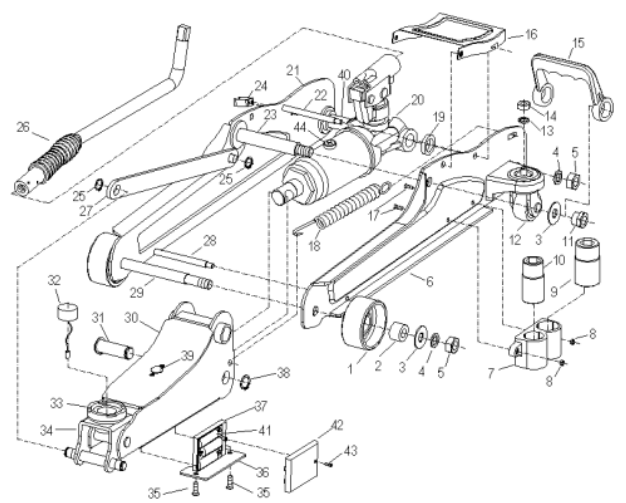

Figure 1 Hydraulic jack explosion figure 
Table 1 The customers' demands of multifunction hydraulic jacks products

\begin{tabular}{|l|l|}
\hline product & Customers' demand \\
\hline \multirow{5}{*}{$\begin{array}{l}\text { multifunction } \\
\text { hydraulic jacks } \\
\text { products }\end{array}$} & Lifting weight \\
\cline { 2 - 2 } & Lifting stroke \\
\cline { 2 - 2 } & Maximum lift height \\
\cline { 2 - 2 } & Minimum lifting height \\
\cline { 2 - 2 } & Car Kits \\
\cline { 2 - 2 } & Lighting \\
\cline { 2 - 2 } & Weight \\
\cline { 2 - 2 } & Service \\
\cline { 2 - 2 } & price \\
\hline
\end{tabular}

In order to facilitate designers to accurately understand and apply the customers' demands, after obtaining the customers' demands of the multifunction hydraulic jacks, we decomposition it into different unit need and establish the demand unit model tree of the multi-function jack.

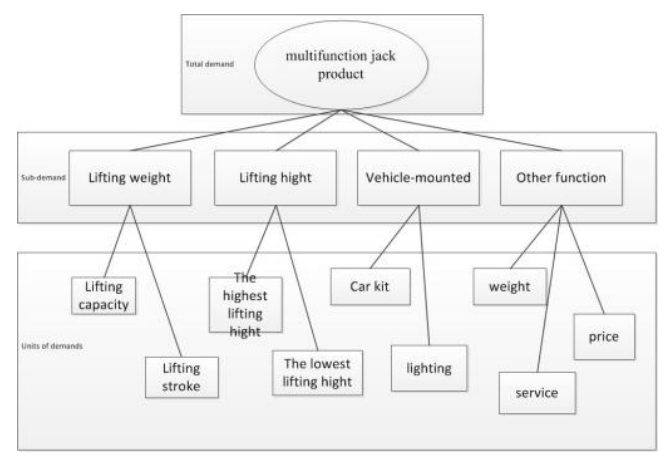

Figure 2 Decomposition of customers' demands of multifunction jack product

\subsection{Determine the weights based on AHP}

Analytic Hierarchy Process, AHP, is a better method of determining the weights. It divides the factors in the complex problems into associated hierarchy, making it become a multi-objective and multicriteria. It is an effective way of quantitative and qualitative analysis.

(1) Construct hierarchy characteristic model

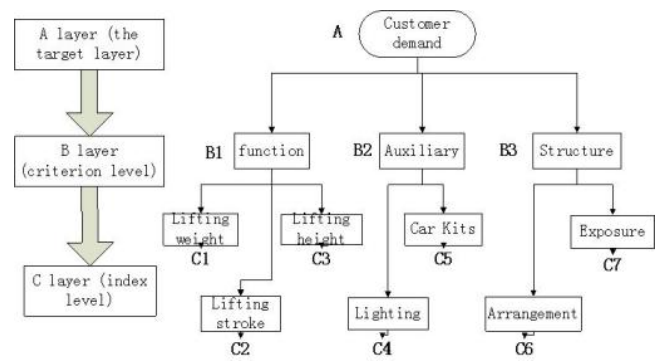

Figure 3 The hierarchical model schematic of jack features

The parts weight distribution properties satisfy the following constraints:

$$
w_{1}+w_{2}+w_{3}=\sum_{k=1}^{K} w_{1 k}+\sum_{l=1}^{L} w_{2 l}+\sum_{h=1}^{H} w_{3 h}=1
$$

(2) Establish judgment matrix

Figure 3 makes the similar character to be hierarchy to illustrate the different level of affiliation between the hierarchical structure and the similar characteristics. Therefore, on this basis you can use a certain scale to compare the relative importance proportion values between similar characteristics.

We assume $B_{1}, B_{2}, \cdots, \mathrm{B}_{\mathrm{n}}$ constitute the next level $\mathrm{B}$ whose purpose are $\mathrm{A}$ level. If we want to analyze the relative importance of $\mathrm{B}_{\mathrm{i}}(1,2, \cdots, n)$ to A, we can construct the judgment matrix below:

$$
\mathbf{J}=\left(\begin{array}{cccc}
B_{1} & B_{2} & \cdots & \mathrm{B}_{\mathrm{n}} \\
u_{11} & u_{12} & \cdots & u_{1 n} \\
u_{21} & u_{22} & \cdots & u_{2 n} \\
\vdots & \vdots & \ddots & \vdots \\
u_{n 1} & u_{n 2} & \cdots & u_{n n}
\end{array}\right) B_{1} B_{2}
$$

In the equation 2 , the elements in the matrix represent the relative importance to the elements in the upper layer. For instance, the value of $u_{n n}$ is the relative importance between $B_{i}$ and $B_{j}$ for A layer. Usually, $u_{i j}$ is $1,2, \cdots, 9$ or their reciprocal.

Judgement matrix $\mathbf{J}=\left[u_{i j}\right] n \times n$ has the following properties:

$$
\begin{aligned}
& \text { (1) } u_{i j}>0, i j=1,2, \cdots, n \\
& \text { (2) } u_{i j}=1 / u_{j i}, i j=1,2, \cdots, n \\
& \text { (3) } u_{i j}=1, i=1,2, \cdots, n
\end{aligned}
$$

Thus $\mathbf{J}$ is positive reciprocal matrix. Therefore, it only needs $n(n-1)^{2}$ determined values to construct judgment matrix.

(3) Hierarchy single sorting and consistency test

The consistency index of calculation of hierarchy single sorting:

$$
C I=\left(\lambda_{\max }-n\right) /(n-1)
$$

In the formula $\mathrm{CI}$ is regarded as the degree of deviation from the consistency. When the consistency proportion $\mathrm{CR}=\mathrm{CI} / \mathrm{RI}<0.1$, it is considered that the result of hierarchy single sorting is satisfied. Otherwise, we need to adjust the judgment matrix to make it possess acceptable consistency. RI is the index of the average random consistency and the relationship between the order of matrix $n(n>2)$ and the RI is revealed as the table 2 . When the matrix order $n<2$, the proportion of consistency CR is 0 . For example, when $n$ is 2 , the judgment $\mathbf{J}$ can be represented as:

$$
\mathbf{J}=\left[\begin{array}{cc}
1 & u_{12} \\
1 / u_{12} & 1
\end{array}\right]
$$

It is easy to know that the max eigenvalue of $\mathbf{J}$ is 2 , and from the equation 3 we know $\mathrm{CI}=0$ and $\mathrm{CR}=0$. 
Table 2 the index distribution of average random consistency

\begin{tabular}{|l|l|}
\hline $\mathrm{N}$ & $\mathrm{RI}$ \\
\hline 1 & 0 \\
\hline 2 & 0 \\
\hline 3 & 0.58 \\
\hline 4 & 0.90 \\
\hline 5 & 1.12 \\
\hline 6 & 1.24 \\
\hline 7 & 1.32 \\
\hline 8 & 1.41 \\
\hline 9 & 1.45 \\
\hline
\end{tabular}

(4) Total sorting and consistency test

Through consistency test of hierarchy single sorting we can deduce $\mathrm{CI}_{\text {total }}=\sum \mathrm{w}_{\mathrm{i}} \mathrm{C}_{\mathrm{Ii}}$,

$$
R I_{\text {total }}=\sum \mathrm{w}_{i} \times R_{I i}, C R_{\text {total }}=C I_{\text {total }} / R I_{\text {total }}
$$

If $\mathrm{CR}_{\text {total }}<0.1$, the result of total sorting reaches the satisfied level of consistency. Otherwise the judgment matrix is needed to be adjusted to reach the satisfied level of consistency.

From the view of function, auxiliary and structure, the table below uses the weight of three layers to carefully divide the customers' demands of jack.

Table 3 the result of calculating the customers' demands weight

\begin{tabular}{|c|c|c|c|c|}
\hline $\mathrm{A}$ & $\mathrm{B}_{1}$ & $\mathrm{~B}_{2}$ & $\mathrm{~B}_{3}$ & $\mathrm{C}_{\mathrm{i}^{*}}$ \\
\hline & 0.593 & 0.249 & 0.157 & \\
\hline $\mathrm{C}_{1}$ & 0.593 & & & 0.352 \\
\hline $\mathrm{C}_{2}$ & 0.249 & & & 0.148 \\
\hline $\mathrm{C}_{3}$ & 0.157 & & & 0.093 \\
\hline $\mathrm{C}_{4}$ & & 0.666 & & 0.166 \\
\hline $\mathrm{C}_{5}$ & & 0.333 & & 0.083 \\
\hline $\mathrm{C}_{6}$ & & & 0.833 & 0.130 \\
\hline $\mathrm{C}_{7}$ & & & 0.166 & 0.026 \\
\hline $\mathrm{CI}_{\mathrm{i}}$ & 0.026 & 0 & 0 & 0.015 \\
\hline $\mathrm{RI}_{\mathrm{j}}$ & 0.520 & 0 & 0 & 0.308 \\
\hline
\end{tabular}

$\mathrm{CR}_{\text {total }}=\mathrm{CI}_{\text {total }} / \mathrm{RI}_{\text {total }}=0.0015 / 0.3087 \leq 0.1$, the total consistency is acceptable.

\subsection{The analysis of similar properties parts}

To describe the physical strength of the interaction of function, auxiliary and structure of different parts, we need to describe in a quantitative way. Table 4 uses the average distribution value in $[0,1]$ to define the fuzzy relationship between two random parts considering each characteristic.

Table 4 the value definition of fuzzy relationship between different parts

\begin{tabular}{|c|c|c|}
\hline number & Type of relaton & The value of interaction \\
\hline 1 & strong & 1.0 \\
\hline 2 & intimate & 0.8 \\
\hline 3 & medium & 0.6 \\
\hline 4 & nomal & 0.4 \\
\hline 5 & weak & 0.2 \\
\hline 6 & nothing & 0 \\
\hline
\end{tabular}

According to the definition of the fuzzy interaction relation of parts in table 4, we can calculate the integrated interactive value:

$$
A=\left\langle\begin{array}{cc}
\sum_{k=1}^{K} A_{1 k}(i, j) w_{1 k}+\sum_{l=1}^{L} A_{2 l}(i, j) w_{2 l}+\sum_{\mathrm{h}=1}^{H} A_{3 h}(i, j) w_{3 h} & i \neq j \\
1 & i=j
\end{array}\right.
$$

In this formula, $A_{1 \mathrm{k}}(i, j), A_{21}(i, j), A_{3 h}(i, j)$ represent the function, structure and auxiliary interactive value based on fuzzy relation; $A(i, j)$ represents integrated interactive value between part $\mathrm{C}_{\mathrm{i}}$ and $\mathrm{C}_{\mathrm{j}}$ after weighting averaging. Therefore we can get the matrix $S$ which contains every

\begin{tabular}{|c|c|c|c|c|c|c|c|c|c|c|c|c|c|c|c|}
\hline & u1 & u2 & u3 & u4 & u5 & u6 & u7 & 148 & ug & u10 & u11 & u12 & u13 & u14 & u15 \\
\hline $\mathrm{u} 1$ & 1 & 0.6006 & 0.6006 & 0.7507 & 0.0628 & 0.7507 & 0.7193 & 0.7507 & 0.1501 & 0.1816 & 0.1501 & 0.0314 & 0.0314 & 0.0314 & 0 \\
\hline u2 & 0.6006 & 1 & 0.4504 & 0.7507 & 0.2130 & 0.6006 & 0.6006 & 0.6006 & 0.1501 & 0.1816 & 0.1501 & 0.0314 & 0.0314 & 0.0314 & 0 \\
\hline u3 & 0.6006 & 0.4504 & 1 & 0.6006 & 0.1501 & 0.6006 & 0.6006 & 0.6006 & 0.4818 & 0.3003 & 0.0314 & 0.3317 & 0.3317 & 0.1501 & 0 \\
\hline $\mathrm{u} 4$ & 0.7507 & 0.7507 & 0.6006 & 1 & 0.1816 & 0.7507 & 0.7507 & 0.7507 & 0.1501 & 0.1816 & 0.1501 & 0.0314 & 0.0314 & 0.0314 & 0 \\
\hline u5 & 0.0628 & 0.2130 & 0.1501 & 0.1816 & 1 & 0.1501 & 0.1501 & 0.1501 & 0.2444 & 0.6320 & 0.7507 & 0.0314 & 0.0314 & 0.0314 & 0 \\
\hline u6 & 0.7507 & 0.6006 & 0.6006 & 0.7507 & 0.1501 & 1 & 0.7507 & 0.7507 & 0.1501 & 0.1816 & 0.1501 & 0.0314 & 0.0314 & 0.0314 & 0 \\
\hline uT & 0.7193 & 0.6006 & 0.6006 & 0.7507 & 0.1501 & 0.7507 & 1 & 0.7507 & 0.1501 & 0.1816 & 0.1501 & 0.0314 & 0.0314 & 0.0314 & 0 \\
\hline u8 & 0.7507 & 0.6006 & 0.6006 & 0.7507 & 0.1501 & 0.7507 & 0.7507 & 1 & 0.1501 & 0.1816 & 0.1501 & 0.0314 & 0.0314 & 0.0314 & 0 \\
\hline u9 & 0.1501 & 0.1501 & 0.4818 & 0.1501 & 0.2444 & 0.1501 & 0.1501 & 0.1501 & 1 & 0.3945 & 0.1257 & 0.7501 & 0.7501 & 0.7501 & 0 \\
\hline u10 & 0.1816 & 0.1816 & 0.3003 & 0.1816 & 0.6320 & 0.1816 & 0.1816 & 0.1816 & 0.3945 & 1 & 0.7501 & 0.0628 & 0.0628 & 0.1571 & 0 \\
\hline u11 & 0.1501 & 0.1501 & 0.0314 & 0.1501 & 0.7507 & 0.1501 & 0.1501 & 0.1501 & 0.1257 & 0.7501 & 1 & 0.0628 & 0.0628 & 0.0628 & 0 \\
\hline u12 & 0.0314 & 0.0314 & 0.3317 & 0.0314 & 0.0314 & 0.0314 & 0.0314 & 0.0314 & 0.7501 & 0.0628 & 0.0628 & 1 & 0.7501 & 0.7501 & 0 \\
\hline $\mathrm{u} 13$ & 0.0314 & 0.0314 & 0.3317 & 0.0314 & 0.0314 & 0.0314 & 0.0314 & 0.0314 & 0.7501 & 0.0628 & 0.0628 & 0.7501 & 1 & 0.7501 & 0 \\
\hline u14 & 0.0314 & 0.0314 & 0.1501 & 0.0314 & 0.0314 & 0.0314 & 0.0314 & 0.0314 & 0.7501 & 0.1571 & 0.0628 & 0.7501 & 0.7501 & 1 & 0 \\
\hline u15 & 0 & 0 & 0 & 0 & 0 & 0 & 0 & 0 & 0 & 0 & 0 & 0 & 0 & 0 & 1 \\
\hline
\end{tabular}
components' relation:

Figure 4 Similarity matrix of jack parts group 


\section{MODULE DIVISION BASED ON FUZZY CLUSTERING ALGORITHM}

When we polymerize the modules, we should make sure that the value of the interaction of function, structure and other parts is maximum. According to the module evaluation matrix based on the equation6, it's hard to directly determine the specific configuration of the module. So we should use the fuzzy clustering algorithm to optimize the parts $(\mathrm{Gu}$, 1999).

With the correlation matrix showing in the figure 4 , we can put the fuzzy similar matrix into fuzzy equivalent matrix by seeking the transitive closure. Then with selecting the appropriate value and letting the value descend, we can form a dynamic clustering map.

The calculation results in total have seven kinds of classification values.

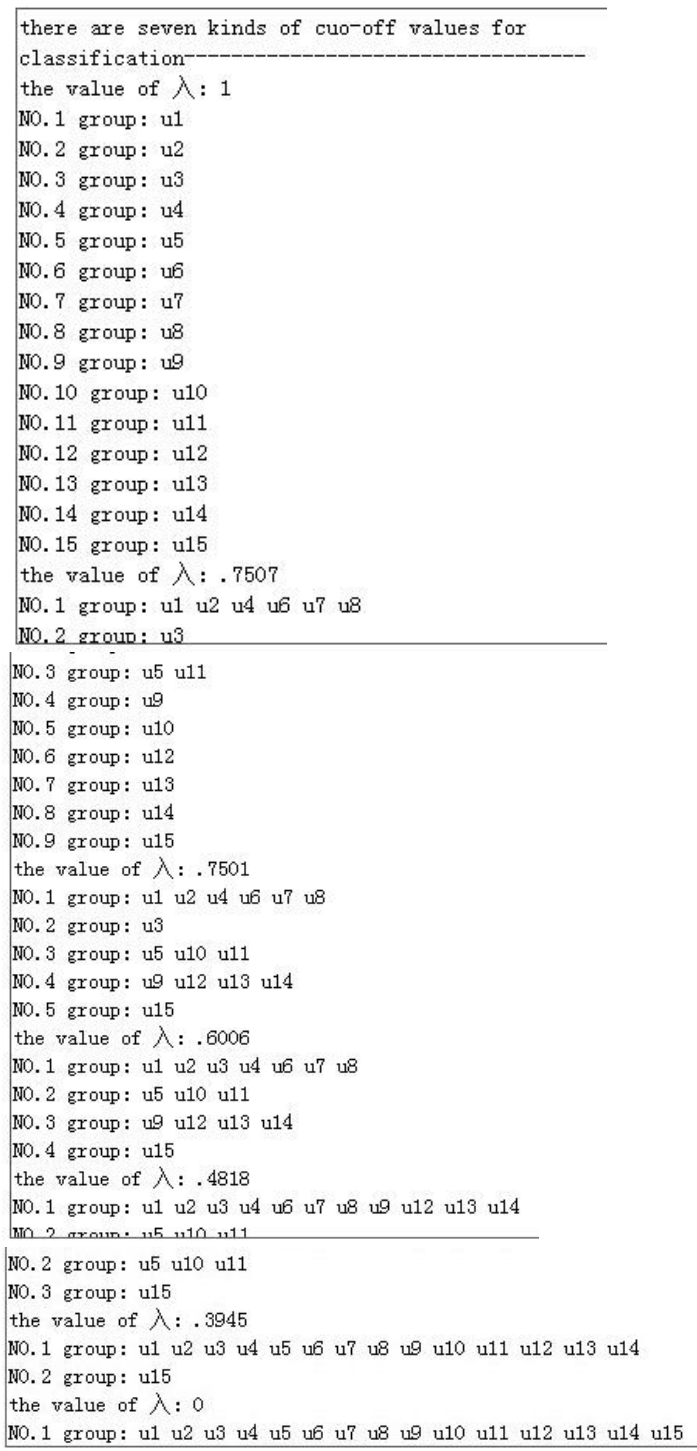

Above all, when $\lambda=0.6006$, the result of classification is more obvious. Here are the groups:

The first category: $\mathrm{u}_{1}, \mathrm{u}_{2}, \mathrm{u}_{3}, \mathrm{u}_{4}, \mathrm{u}_{6}, \mathrm{u}_{7}$, $\mathrm{u}_{8}$ (hydraulic group, heart tube group, handle tube set, piston set, switch back to the oil group, regulator bolt group, outgassing switch group)

The second category: $\mathrm{u}_{5}, \mathrm{u}_{10}, \mathrm{u}_{11}$ (top seat group, rocker group, the top plate group)

The third category: $\mathrm{u}_{9}, \mathrm{u}_{12}, \mathrm{u}_{13}, \mathrm{u}_{14}$ (stent group, rear wheel, rear wheel fork welding group, bearing plate group)

The fourth category: $\mathrm{u}_{15}$ (electric group)

\section{CONCLUSION}

First of all, by using the method of AHP analyze, the article decomposes the customers' demands, calculates the weight of each decomposition unit and quantifies the different customers' demands which impact the foundation of correlation matrix; then with using the fuzzy clustering algorithm, it make components into module. Fuzzy clustering algorithm does not need to build complex objective function or ensures the initial value, nor does it have too many constraints. So it is a simple and comprehensive way to design the modular product.

\section{REFERENCES}

[1] DONG Zhong Yuan \& Jiang Ke Zhu. Design Methodology. Bei Jing:Higher Education Press,1991

[2] Gu P, Sosale. Product modularization for life engineering. Robotics and Computer Integrated Manufacturing, 1999, 15(5):387-401

[3] GAO Fei, XIAO Gang, PAN Shuangxia, et al. Method of product function module partition. Chinese Journal of Mechanical Engineering, 2007, 43(5):29-35 (in Chinese).

[4] SUN Wei. Integrated design system architecture for mass customization production mode and its key technology research. DaLian: Dalian University of Technology, 2000

[5] STONE R B, WOOD K L, CRAWFORD R H.A heuristic method for identifying modules for product architectures. Design Studies, 2000, 21(1):5-31.

[6] Tan Yan Hong, Dan Bin, Zhang Xu Mei. Product configuration based on modular product family and conversion of customer demand. Industrial Engineering and Management, 2008, 1.

[7] Zhu Bin \& Wang Zhan \& Yang Hai Ping. Product configuration based on uncertain customers' demands. Aeronautical Manufacturing Technology, 2008, 5: 73-82.

Figure 5 Classification result 